\title{
DIFFRACTION IMAGING AND IMAGE PROCESSING - TOOLS FOR LOCALIZATION AND CHARACTERIZATION OF FRACTURED ZONES BY SEISMIC DATA
}

\author{
MAXIM I. PROTASOV ${ }^{1}$ AND YAROSLAV V. BAZAIKIN ${ }^{2}$ \\ ${ }^{1}$ Institute of Petroleum Geology and Geophysics \\ 3, Koptuyg st., Novosibirsk, Russia 630090 \\ protasovmi@ipgg.sbras.ru,www.ipgg.sbras.ru \\ ${ }^{2}$ Institute of Petroleum Geology and Geophysics \\ 3, Koptuyg st., Novosibirsk, Russia 630090 \\ bazaikinyv@ipgg.sbras.ru,www.ipgg.sbras.ru
}

Key words: Diffraction imaging, DFN model, topological analysis, seismic data.

\begin{abstract}
The paper presents a technique for the localization and characterization of fractured zones by seismic data. The developed approach combines the diffraction imaging and topological analysis of diffraction images. The testing results for realistic synthetic models and real seismic data demonstrate the possibility of a reliable restoration of the fractured zones' statistical characteristics.
\end{abstract}

\section{INTRODUCTION}

The ability to locate fractures precisely and to characterize their properties is of high importance. Various algorithms exist to discover these microstructures through the analysis of diffracted/scattered elastic waves. The paper presents an application of the new method (Protasov et al., 2019) to the realistic synthetic and real data and describes it from the practical point of view. The approach is a combination of two techniques to provide recovery of fracture characteristics from seismic data.

First, we develop a statistical model that adequately describes fracture systems on the seismic scale. The main existing approaches to statistical modeling of fracture systems are described (Xu and Dowd, 2010). Explicit statistical modeling of fracture networks and the medium permeability evaluations were carried out, for example, in (Odling et al. 2004). Here, we use a discrete fracture network (DFN) concept that requires an explicit spatial position, size, and orientation for each fracture (Xu and Dowd, 2010). Thus, the fractures' attributes (i.e., direction and size) are defined by corresponding statistical distributions.

Then, for a seismic model that contains modeled fractures, we provide the diffraction imaging. We use 3D diffraction imaging workflows for fracture detections that include two main procedures: the prestack asymmetric migration procedure, a weighted data summation, and image spectral decomposition (Protasov et al., 2016, 2019).

To obtain the information about the fracture parameters at the second stage, we propose an 
original topological analysis of the diffraction images that contain fractured zones. The basis for such an analysis provides the observation that different amplitude levels of diffraction images give topologically different objects. In this situation, computational topology algorithms (Bazaikin et al., 2013) extract information about those objects.

We present and discuss the numerical results for the synthetic model. Finally, the application to $3 \mathrm{D}$ real data is provided together with a focus on practical aspects.

\section{DFN MODELING: SEISMIC SCALE}

For the statistical modeling of a fracture system, we used the general scheme described in (Xu et al., 2010). Fractures are represented in the form of ellipsoids. The ellipsoid plane's orientation is determined by the direction of the normal parallel to the smaller axis of the ellipsoid. For all the models considered, the dip angle's average value is chosen equal to pi / 2 , i.e., fractures are vertical. The average value of the dip direction and the variance of the deviation from this direction are selected separately for each fracture family. The fractures' geometric dimensions are determined by the dimensions of the main axes (or semi-axes). Following (Xu et al., 2010), the large axis (fracture length) L is modeled statistically. The mean axis (fracture width) W is determined from the simulated fracture length by specifying the statistical distribution L/W. The value of the smaller axis (fracture thickness) $\mathrm{T}$ is given as a constant. After modeling the fractures' geometry, the next step is to put the model on a coarse grid. The complexity of the problem is that the fractures thickness is uswally much smaller than the grid step. For each cell using statistical modelin. computed on a sparse

3 DIFTRACTION IM
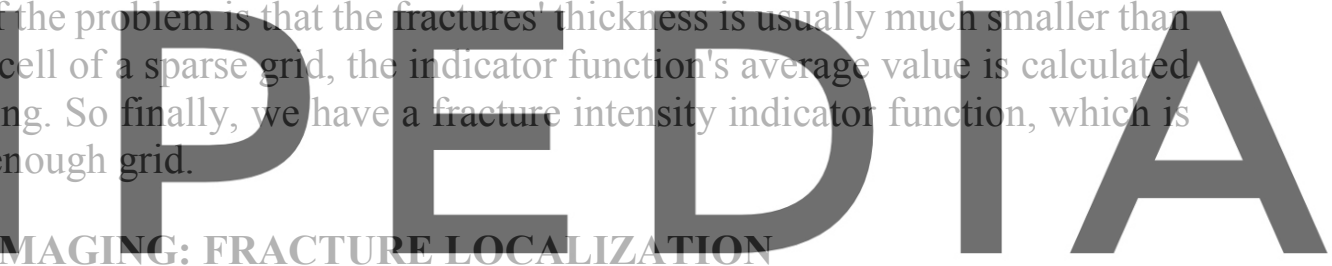

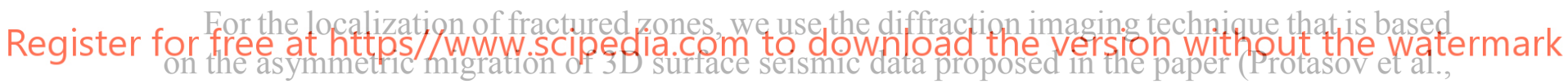
2017):

$$
\begin{aligned}
& \operatorname{Image}(\mathbf{x} ; \mathrm{az}, \beta)=\int \tau_{p}^{g b_{2}}\left(x_{s}, y_{s} ; \mathbf{x}, \gamma, \theta, a z, \beta ; \omega\right) \mathbf{T}_{\mathrm{p}}^{g b_{1}}\left(x_{r}, y_{r} ; \mathbf{x}, \gamma, \theta, a z, \beta ; \omega\right) \\
& \operatorname{data}\left(x_{r}, y_{r} ; x_{s}, y_{s} ; \omega\right) k(\mathbf{x}, \gamma, \theta, \beta) d x_{r} d y_{r} d x_{s} d y_{s} d \omega d \gamma d \theta
\end{aligned}
$$

Here Image $(\mathbf{x} ; \mathrm{az}, \beta)$ is the recovered function, data $\left(x_{r}, y_{r} ; x_{s}, y_{s} ; \omega\right)$ is multicomponent surface seismic data in the frequency domain, and $\tau_{g b p}^{s}\left(x_{s}, y_{s} ; \omega ; \alpha, \theta, a z, \beta\right), \mathbf{T}_{g b p}^{r}\left(x_{r}, y_{r} ; \omega ; \alpha, \theta, a z, \beta\right)$ is normal derivatives of Gaussian beams and their potentials at the source positions. The beams are computed by ray tracing from every image point (see Figure 2a), and their derivatives depend on the structural dip, azimuth, and opening angles (see Figure $2 \mathrm{~b}$ ).

The left-hand side of the imaging formula (1) determines the 3D spatial Fourier transform of the unknown function $f(\mathbf{x} ; \mathrm{az}, \beta)$ followed by its quasi-inverse: 


$$
\operatorname{Image}(\mathbf{x} ; \mathrm{az}, \beta)=\iiint_{X_{p a r}} e^{i \cdot \mathbf{x} \cdot \mathbf{x}} F(\omega(\mathbf{p})) d \bar{p} \iiint_{X} e^{-i \cdot \mathbf{p} \cdot \mathbf{y}} \cdot f(\mathbf{y} ; a z, \beta) d \mathbf{y}
$$

Here, $f=\lambda_{1}+2 \mu_{1} \cos ^{2}(2 \beta)+v_{0}^{p^{2}} \rho_{1} \cos (2 \beta)$. This is not the Fourier transform's exact inversion because it is performed not over the whole phase space but only over its subdomain Xpar (the set of partial reconstruction, see Protasov et al., 2016). This subdomain is a circular sector that is defined by the frequency bandwidth $\left[\omega_{1}, \omega_{2}\right]$ of the source function and the available range of structural dip and azimuth angles $\left[\alpha_{1}, \alpha_{2}\right],\left[\theta_{1}, \theta_{2}\right]$ (see Figure 3 ):

The structure of the partial reconstruction set, defined by (3), lies at the heart of the proposed method of imaging small scale heterogeneities. The main principles are explained as follows: by changing opening and the dip and azimuth angles of $\left[\alpha_{1}, \alpha_{2}\right],\left[\theta_{1}, \theta_{2}\right]$, respectively, one changes the structure of the set of partial reconstruction and, thus, controls the geometry of visible and invisible elements of the geological cross-section. Specifically, any small scale (small scale) object such as a diffractor/scatterer, crack, fault, pinch, and so on, possesses an extended spatial spectrum and, thus, will be presented for a wide range of partial reconstruction sets. In contrast, any regular interface possesses a very narrow spatial spectrum. Therefore, one can easily choose the Gaussian beams' geometry that provides the partial reconstruction set without this spectrum (Protasov et al., 2017, 2019).

The critical point for successful diffraction imaging is the ratio of the scattered waves' amplitudes to the reflected waves' amplitudes. For the proposed diffraction imaging approach,
this ratio can achieve minimal values and varies down to $1 \%$; however, the image quality is
still acceptable (Protasor et al, 2019).
4 TOPOLOGICAL ANALYSIS: FRACTURE CHARACTERILATION

Let the function $f: U \rightarrow \mathbb{R}$ defines the diffraction image's amplitude field, where $U$ - a 3D

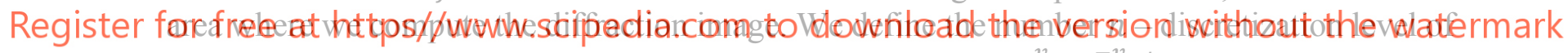
the values of the function $f$. We define values $a_{i}=v_{\min }+i \frac{v_{\max }-v_{\min }}{n}, i=0, \ldots, n$, where $v_{\min }$ and $v_{\max }$ minimum and maximum values of the function $f$. Then we consider a set of excursion sets $M_{i}=\left\{(x, y, z) \mid f(x, y, z) \leq a_{i}\right\}, i=0, \ldots, n$. It is evident that excursion sets are nested inside each other: $M_{0} \subset M_{1} \subset \cdots \subset M_{n}$. Therefore we can construct the absorption tree $\Gamma=\Gamma(f)$ (for more details, see Bazaikin et al., 2013). The geometric characteristics of each connected component of each excursion set are stored in the fields of the corresponding vertex of the absorption tree $\Gamma$. We postulate that the leaves of the tree $\Gamma$ correspond to the original fractures or set of fractures. To calculate the fracture's geometric characteristics (or fractures set) corresponding to the leaf $u$, we find its support vertex $v$. Then, the characteristics of the vertex $\mathrm{v}$ will be the characteristics of the corresponding fracture.

The main problem in applying real data is "topological noise," and merge trees are useful tools to do this. We use three ways to eliminate topological noise and collect the most helpful information from the merge tree. At first, we choose a typical amplitude interval, which is of most importance for physical reasons. Secondly, we erase the shortest branches of the merge tree corresponding to the noise of amplitude data. At third, we erase those branches which 
correspond to fractures with too small volumes. We need to find a critical parameter of noise elimination related to a particular geophysical situation in every way.

\section{SYNTHETIC DATA EXAMPLE}

For seismic scale fracture modeling, a cluster model for the distribution of fractures is realized. At the first stage, families of large fractures (or fracture corridors) are modeled. Next, in the vicinity of each large fracture center of small fractures and their remaining geometric parameters are generated. Figure 1a presents the model's example with two families of large fractures: 80 large fractures from the first family and 120 fractures from the second family. The random coordinates of the centers of large fractures were generated independently and uniformly in the calculated region.

In Figure 1, diffraction images are also presented. They were got for the range of dip angles $\left[\begin{array}{ll}10^{\circ} & 50^{\circ}\end{array}\right]$ but the different range of azimuth angles: $\left[\begin{array}{lll}0 & 360^{\circ}\end{array}\right]$ (Figure 1b), $\left[\begin{array}{cc}-30^{\circ} & 60^{\circ}\end{array}\right]$ and

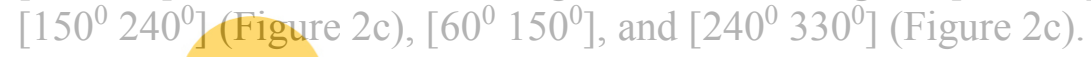
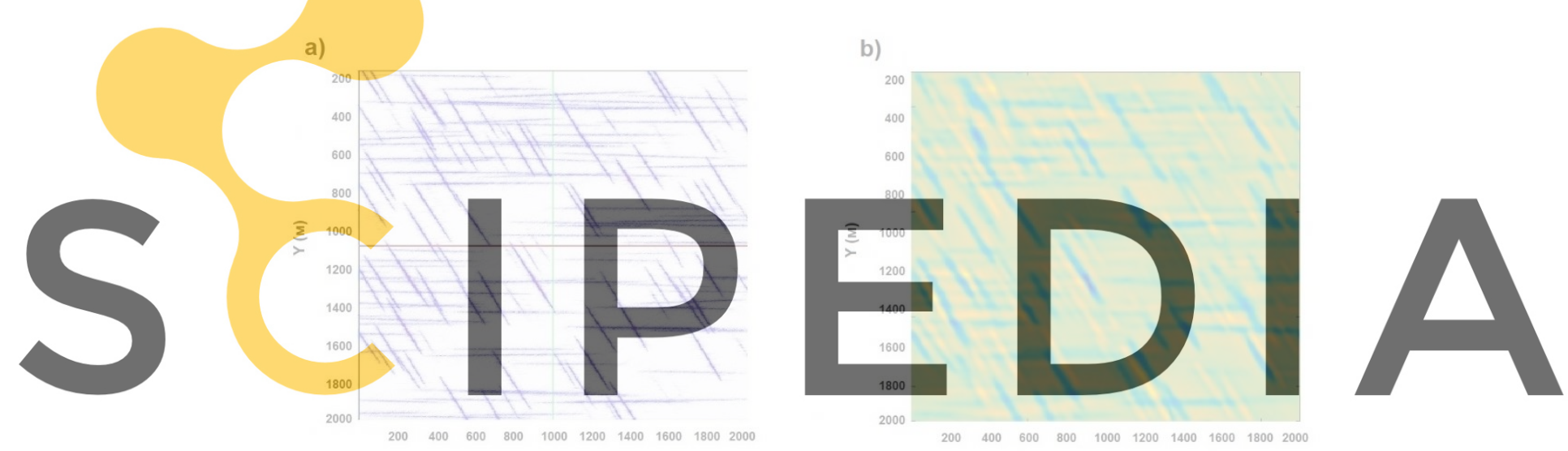

Register for free at https//wwwxscipedia.com to download the verssion without the watermark
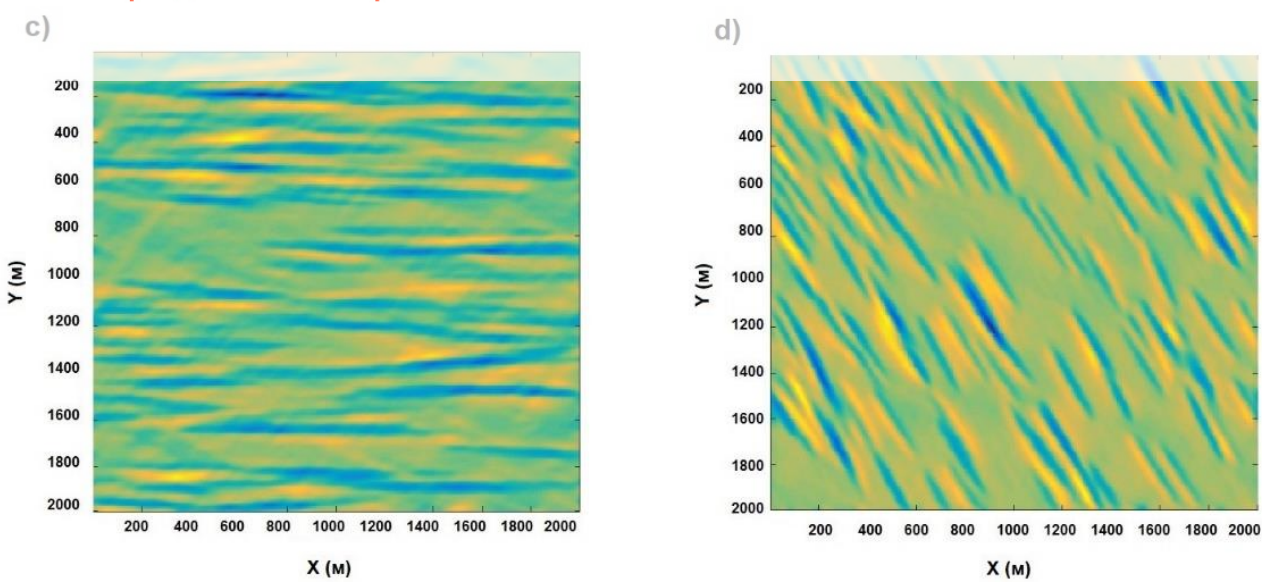

Figure 1: DFN seismic model (a) and Diffraction images in the XY plane $(\mathrm{Z}=2500 \mathrm{~m})$ : $\mathrm{b}$ - the sum of selective images for all azimuth angles; $\mathrm{c}-$ the sum of selective images for the sector of azimuth angles $\left[-30^{0}, 60^{0}\right]$ and $\left[150^{\circ}, 240^{\circ}\right] ; \mathrm{d}-$ the sum of selective images for the sector of azimuth angles $\left[60^{\circ}, 150^{\circ}\right]$ and $\left[240^{\circ}, 330^{0}\right]$. 
Figure 2 presents the original model with 2 families of large fractures (Fig. 2a) and the constructed models (Fig. 2b, 2c, 2d) using fracture characteristics recovered from the corresponded diffraction images (Fig. 1b, 1c, 1d) by the described topological algorithm. One can visually observe wrong fracture reconstruction for the full diffraction image. Still, if the fracture families are differentiated on the images, then the topological algorithm recovers characteristics that give a similar picture. Moreover, most of these characteristics are statistically equivalent to the original model's characteristics (see Table 1). The number of recovered fracture corridors is less than the number of fracture corridors in the model. It means that recovery is not identical; however, the statistical distribution of the modeled fracture corridors and the recovered ones are very close for each of the two families. Also, the modeled and recovered fracture corridors' average length values are very close, and the average directions of the main axis are almost the same.
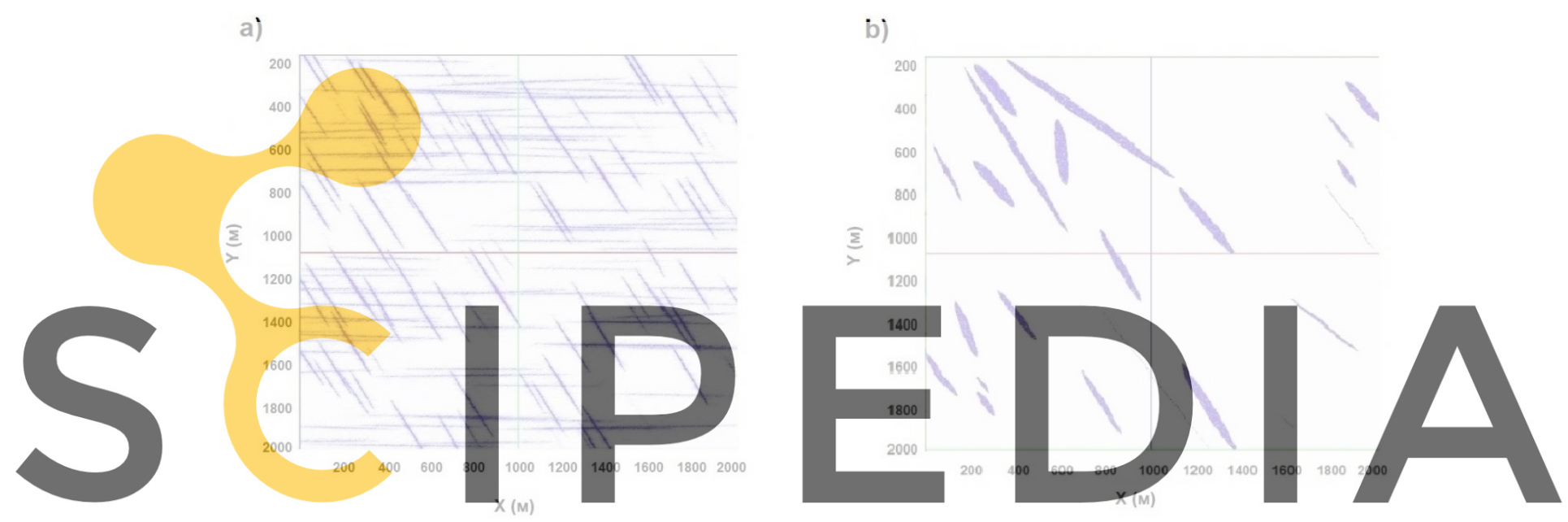

Register for ffee at https//www.scipedia.com to download the version without the watermark
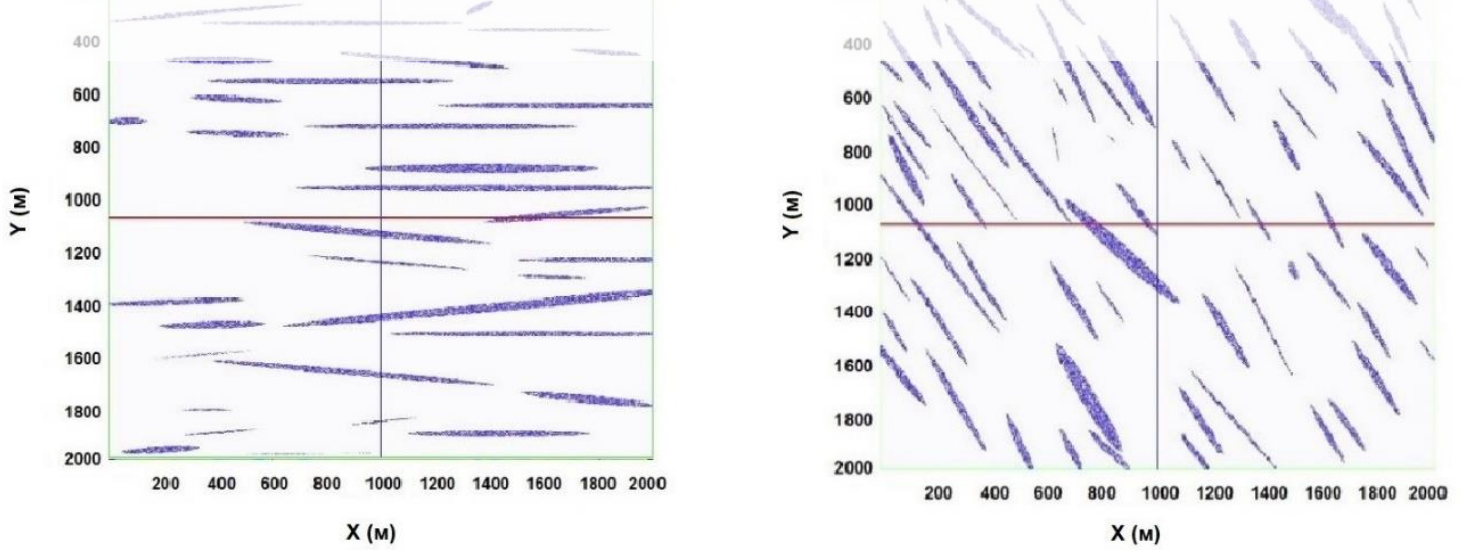

Figure 2: DFN seismic model (a) and models recovered from diffraction images in the $X Y$ plane $(\mathrm{Z}=2500 \mathrm{~m}): \mathrm{b}$ - from the diffraction image with all azimuth angles; $\mathrm{c}$ - from the diffraction image with azimuth angles $\left[-30^{0}\right.$, $\left.60^{0}\right]$ and $\left[150^{\circ}, 240^{\circ}\right] ; \mathrm{d}-$ from the diffraction image with azimuth angles $\left[60^{\circ}, 150^{\circ}\right]$ and $\left[240^{0}, 330^{\circ}\right]$. 
Table 1: The true characteristics and recovered characteristics from the diffraction images.

\begin{tabular}{ccccc}
\hline Model & $\begin{array}{c}\text { Average } \\
\text { length }\end{array}$ & \multicolumn{2}{c}{ Average direction } & $\begin{array}{c}\text { Number of } \\
\text { fractures }\end{array}$ \\
\hline DFN family 1 & 401.33 & $(0.8669$, & $0.4984,-0.0008)$ & 120 \\
\hline $\begin{array}{c}\text { DFN from image } \\
\text { on the Figure 1d }\end{array}$ & 405 & $(0.8673$, & $0.4978,-0.0048)$ & 83 \\
\hline DFN family 2 & 1000.4 & $(0.0030$, & 1.0000, & $0.0046)$ \\
\hline $\begin{array}{l}\text { DFN from image } \\
\text { on the Figure 1c }\end{array}$ & 890 & $(0.0070$, & $1.0000,-0.0018)$ & 56 \\
\hline
\end{tabular}

\section{REAL DATA EXAMPLE}

We proyide real data study for the area where the diffraction imaging algorithm identifies a fractured zone (Protasov et al., 2019). It is shown that for typical practical situations, when the scattered waves' amplitudes are significantly lower than the amplitudes of the reflected waves, the total migrated image does not give visible objects of diffraction. On the contrary, fracture zones are visible in the diffraction images. Moreover, if the ratio of the scattered and reflected waves' amplitudes is not less than 10\%. The diffraction image does not contain any other visible

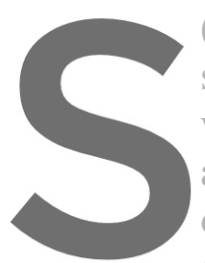
(i.e., comparable amplitude) objects. residual reflections, suppose the ratio of the within $1 \%$, in the diffraction image, in addition to are visible (their amplitude is of the same ord object). In this case, thes approximately $1 \%$ of the most migration procedures.

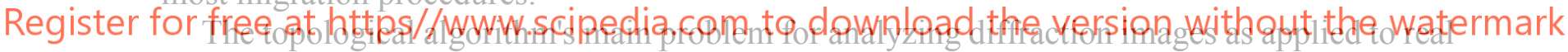
data is "topological noise." In many ways, the appearance of such noise is due to an insufficientily good signal-to-noise ratio. In this case, the signal is diffracted/scattered waves, so it is challenging to achieve a high signal-to-noise ratio on real data. However, the author's algorithm for constructing diffraction images gives entirely satisfactory results, at least in this example. But the topological noise remains, so three methods are used to eliminate it and extract the most useful information from the absorption tree. First, a typical range of amplitudes is selected, which is the most important from physical considerations. Second, the shortest branches of the absorption tree corresponding to the amplitude data's noise are cut off. Thirdly, those branches that correspond to cracks with too small volumes are cut off. A critical parameter for noise elimination is found in all methods, which is associated with a specific geophysical situation.

Then we describe these methods in more detail below. Let us consider the function $f$ as the amplitude of a three-dimensional diffraction image $X$. Then $f$ is represented by values in each elementary volume $X$. Let us define $f_{\min }$ and $f_{\max }$ the minimum and maximum values of the amplitudes $\mathrm{X} \Delta \mathrm{f}=\mathrm{f}_{\text {max }}-\mathrm{f}_{\text {min }}$. The first parameter we used was the filtering level $\mathrm{N}$. Further, 
for each, $i=0,1, \ldots, N$ we introduce $X_{i}$, which should consist of those elementary volumes $v$ for which $\mathrm{f}(\mathrm{v}) \leq \mathrm{f}_{\min }+\Delta \mathrm{f} \cdot \mathrm{i} / \mathrm{N}$. It is clear that a higher filtration level gives a more detailed picture of the function levels' topological properties $\mathrm{f}$.

To eliminate noise with negligible amplitude, we must select a threshold parameter Amp crit from $0 \%$ to $100 \%$. Then we need to shrink the absorption tree, i.e., glue all vertices (respectively, all branches) belonging to the same level i, if $100 \cdot(\mathrm{N}-\mathrm{i}) / \mathrm{N} \leq \mathrm{Amp}_{\text {crit }}$. The rest of the tree structure remains unchanged. This procedure assumes that all branches with an endvertex amplitude greater than $\mathrm{f}_{\max }-\Delta \mathrm{f} \cdot \mathrm{Amp}_{\text {crit }} / 100$. If we consider each branch of the absorption tree as a fracture, we remove those fractures whose minimum amplitude values are

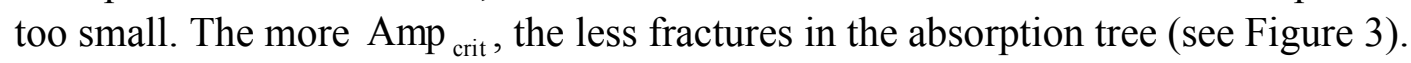
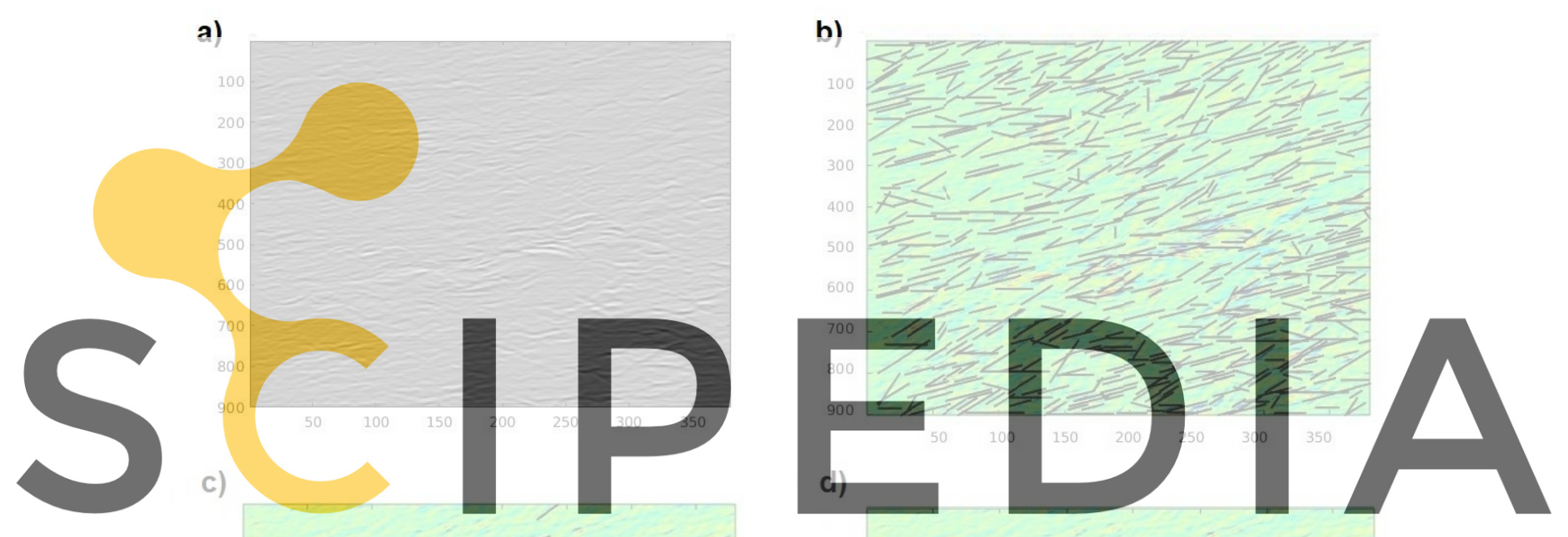

Register for free at https//www.scipedia.com
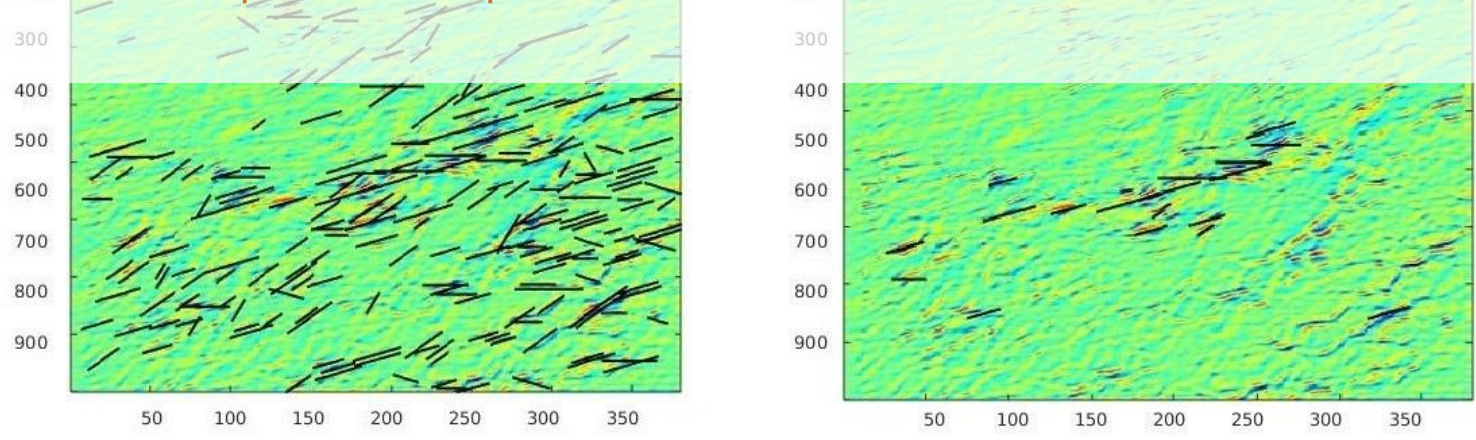

Figure 3: Fractures extracted from the same image (a) with different threshold values of the amplitude: $=50 \%$ (b), $70 \%$ (c), and $90 \%(d)$.

The number of elementary volumes measures the last parameter of noise reduction. We delete all the absorption tree nodes with the volume of connectivity components less than Vol with all the branches containing such nodes. The more Vol, the less cracks in the absorption tree (see Figure 4). 
a)

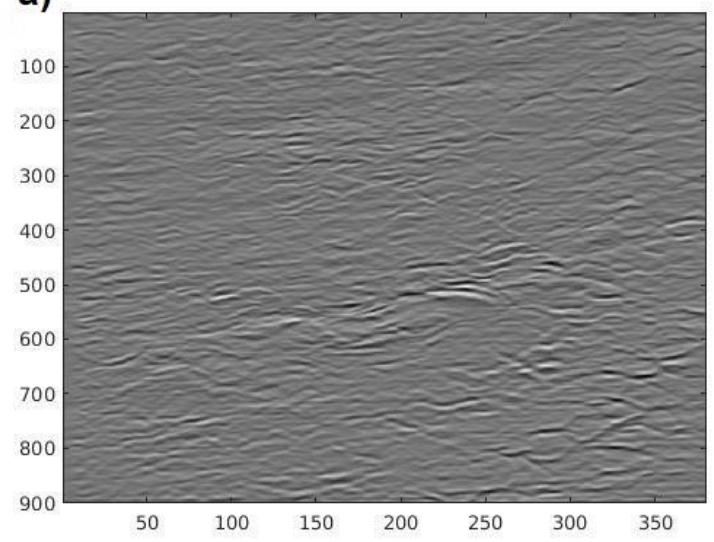

c)

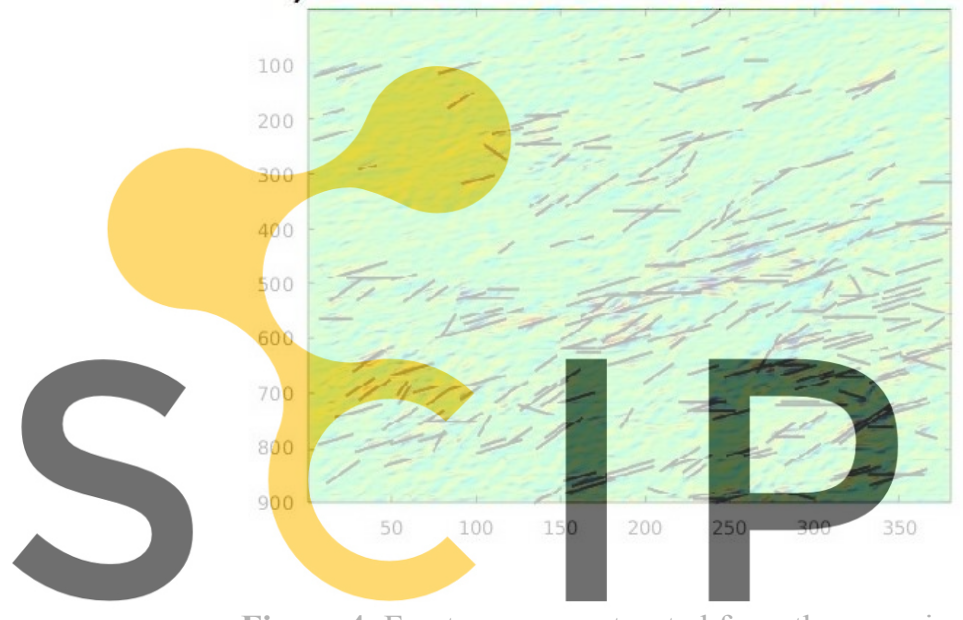

b)

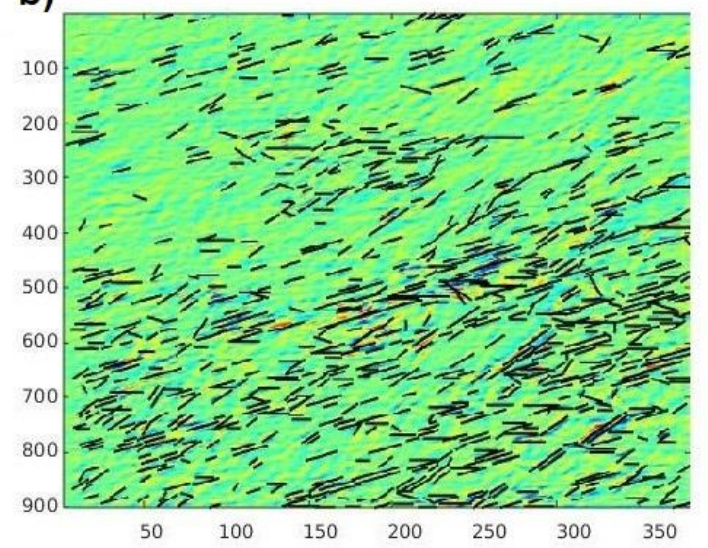

d)

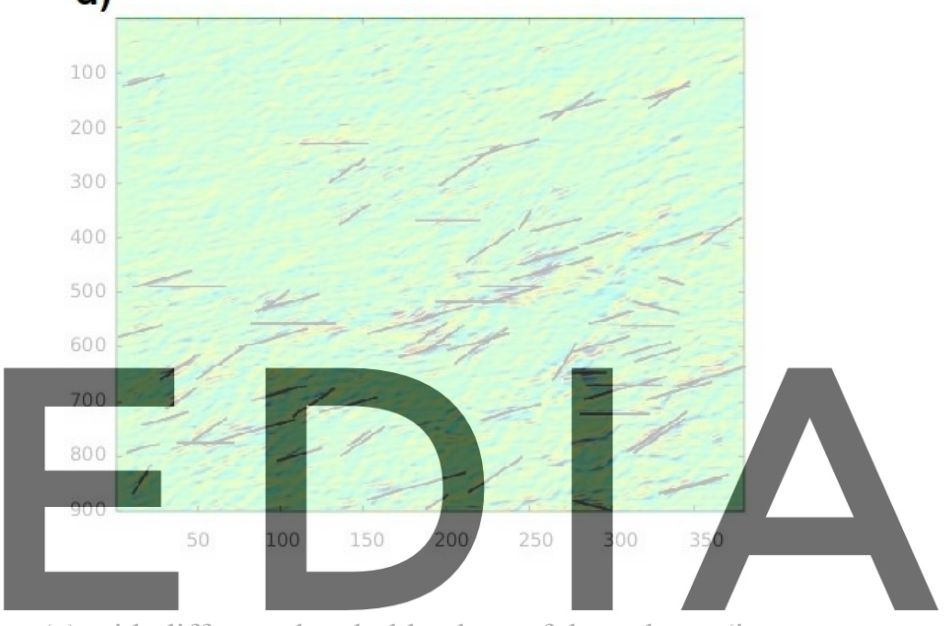

Figure 4: Fractures reconstructed from the same image (a) with different threshold values of the volume (in

Register for free at https//www.scipedia.com to download the version without the watermark

In the synthetic data study, we determine the necessity to give an azimuthal decomposition of the full diffraction image. Analyzing azimuthal diffraction images and their sum, we divide them into four sectors (Figure 5, left): vicinity of $0^{\circ}$, vicinity of $45^{\circ}$, vicinity of $90^{\circ}$, and $135^{\circ}$. Then we apply the described above topological algorithm to each azimuthal image. As we mentioned above, the main problem in application to real data is "topological noise." So, we provide the described strategy to provide a stable and reliable solution. The result of the application one can observe on the right of Figure 5. The black lines are the recovered fractures. One can observe the correspondence between image, i.e., amplitudes distribution, and the recovered fractures, i.e., black lines. We underline that there are three fractures families with a rather significant number, while one more (vicinity of $90^{\circ}$ ) is not representative. Therefore, we think that these three families' statistical parameters are appropriately reconstructed (the strong argument is the synthetic example) and can be used for fracture modeling. Simultaneously, the 4th one (vicinity of $90^{\circ}$ ) should be calibrated better (Table 2). Though other families are representative, their parameters should also be calibrated by the well data. 
a)

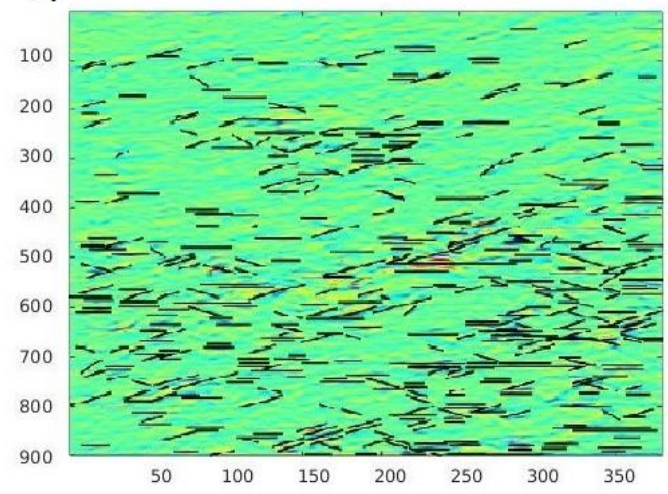

c)

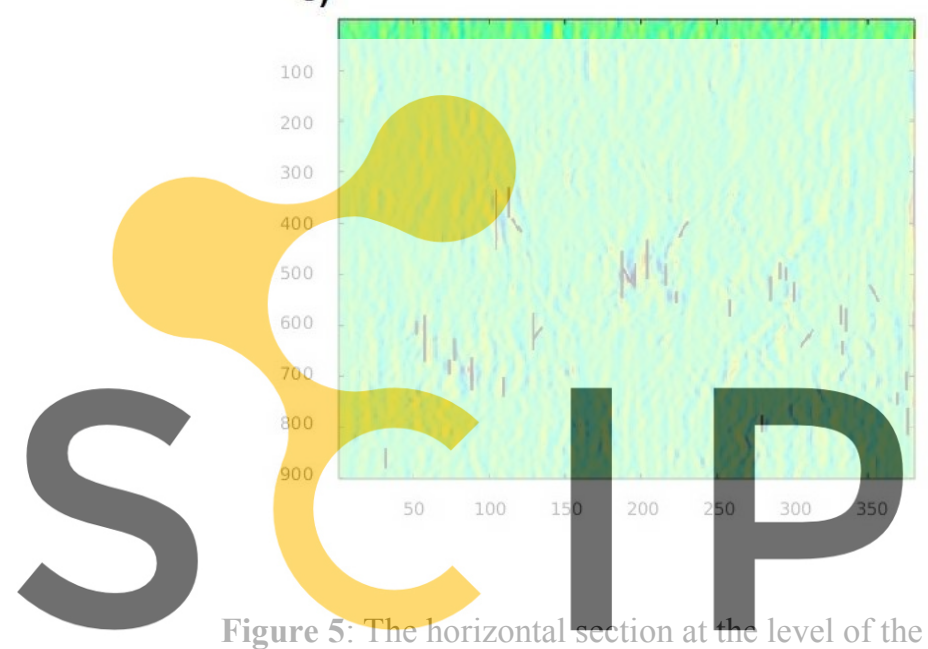

b)

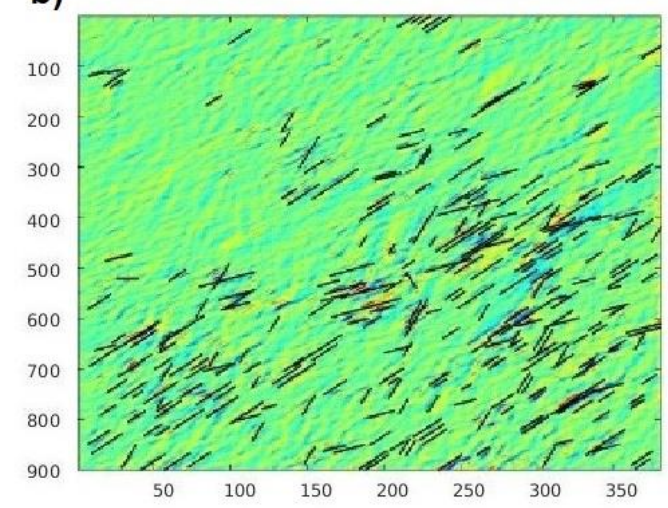

d)

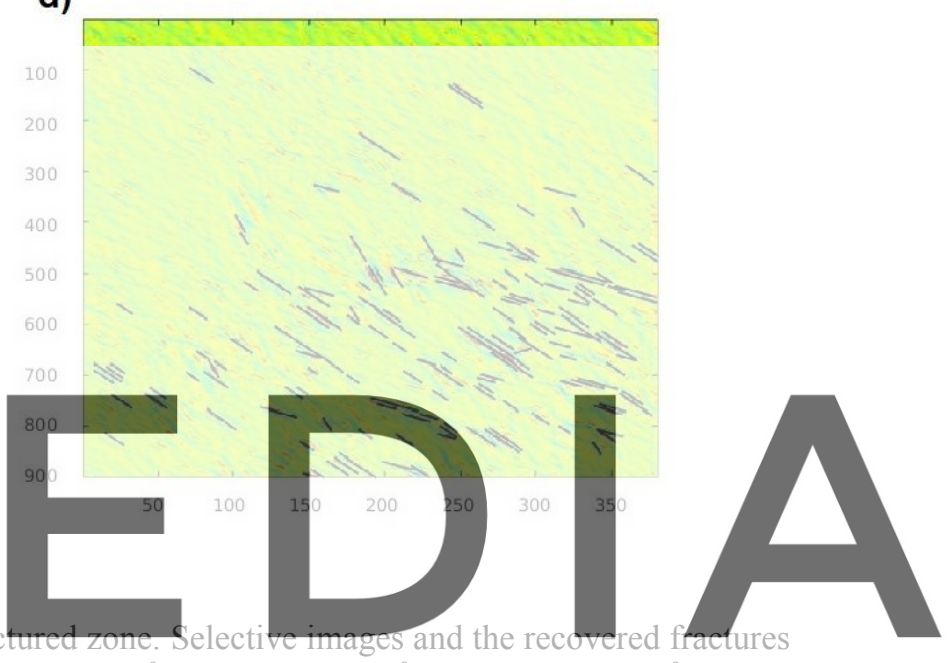

(black lines) for sectors: a) vicinity of $0^{\circ}$, b) vicinity of $45^{\circ}$, c) vicinity of $90^{\circ}$, d) vicinity of $135^{\circ}$

Register for free at https//www.scipedia.com to download the version without the watermark

Table 2: The fracture characteristics recovered from the images decomposed by azimuthal sectors.

\begin{tabular}{cccc}
\hline Model & $\begin{array}{c}\text { Average } \\
\text { length }\end{array}$ & Average azimuth direction & $\begin{array}{c}\text { Number of } \\
\text { fractures }\end{array}$ \\
\hline $\begin{array}{c}\text { DFN from image } \\
\text { vicinity of } 0^{0}\end{array}$ & $500 \mathrm{~m}$ & $1^{0}$ & 2493 \\
\hline $\begin{array}{c}\text { DFN from image } \\
\text { vicinity of } 45^{0}\end{array}$ & $499 \mathrm{~m}$ & $23^{0}$ & 1164 \\
\hline $\begin{array}{c}\text { DFN from image } \\
\text { vicinity of } 90^{0}\end{array}$ & $668 \mathrm{~m}$ & $70^{0}$ & 191 \\
\hline $\begin{array}{c}\text { DFN from image } \\
\text { vicinity of } 135^{0}\end{array}$ & $469 \mathrm{~m}$ & $178^{0}$ & 469 \\
\hline
\end{tabular}

\section{CONCLUSIONS}

The paper has presented a combination of diffraction imaging and topological analysis of the diffraction images that recover the fractured zone and their characteristics. The numerical 
results on synthetic data show a reasonable reconstruction of the fracture corridors' statistical distribution, an average value of the fracture corridors length, and an average value of the fracture corridors directions. The numerical results on the real data show that the described algorithmic combination can reconstruct the fracture zone and characterize them. Better confirmation and more arguments about the practical application of the approach could be obtained by introducing well data, i.e., core analysis and FMI data.

\section{ACKNOWLEDGMENTS}

The reported study was funded by RFBR and GACR, project number 20-55-26003.

\section{REFERENCES}

[1] Bazaikin, Ya.V., Baikov, V.A., Taimanov I.A., Yakovlev A.A., Numerical analysis of topological characteristics of three-dimensional geological models of oil and gas fields. Math. Modeling (2013) 25(10):19-31. (in Russian)

[2] Odling, N.E., Harris, S.D., Knipe, R.J. Permeability scaling properties of fault damage zones in siliclastic rocks. Journal of Structural Geology (2004) 26:1727-1747.

[3] Protasov, M.I., Tcheverda, V.A., Reshetova, G.V. Fracture detection by Gaussian beam imaging of seismic data and image spectrum analysis. Geophysical Prospecting (2016) 64(1): $68-82$.

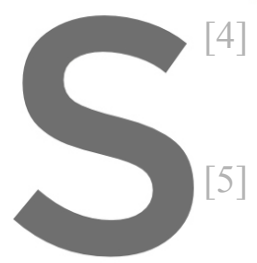

4] Protasov, M,I., Gad of fault and frac Geophysical prosp

Protasov, M.I., Khachkov fractured zones vil (2019) 84(5):093-0102.
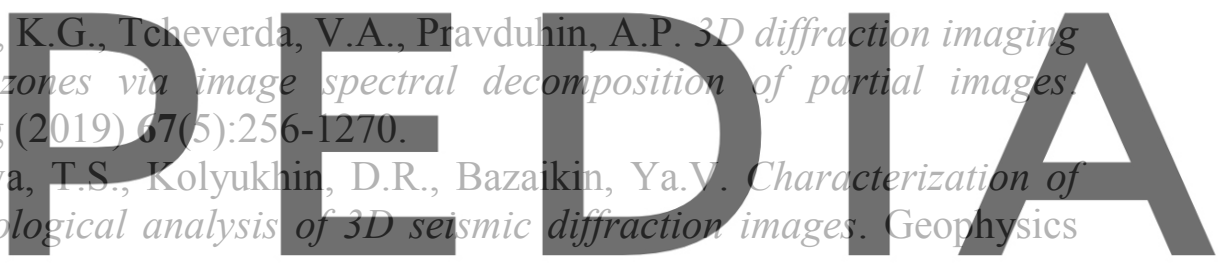

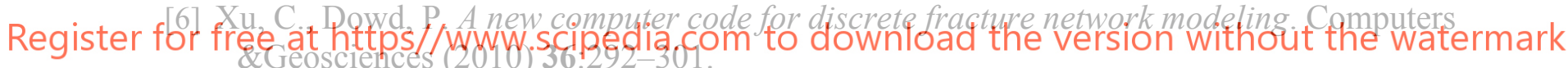

